\title{
How the threshold of a neuron determines its capacity for coincidence detection
}

\author{
Richard Kempter $^{\mathrm{a}, *}$, Wulfram Gerstner ${ }^{\mathrm{b}}$, J. Leo van Hemmen ${ }^{\mathrm{a}}$ \\ a Physik Department der TU München, D-85747 Garching bei München, Germany \\ b Swiss Federal Institute of Technology, Center of Neuromimetic Systems,EPFL-DI, CH-1015 Lausanne, Switzerland
}

\begin{abstract}
Coherent oscillatory activity of a population of neurons is thought to be a vital feature of temporal coding in the brain. We focus on the question of whether a single neuron can transform a spike code into a rate code. More precisely, how does a neuron vary its mean output firing rate, if its input changes from random to coherent? We investigate the coincidence detection properties of an integrate-and-fire neuron in dependence upon internal parameters and input statistics. In particular, we show how coincidence detection depends on the membrane time constant and the threshold. Furthermore, we demonstrate that there is an optimal threshold for coincidence detection and that there is a broad range of near-optimal threshold values. Fine-tuning is not necessary. (C) 1998 Elsevier Science Ireland Ltd. All rights reserved.
\end{abstract}

Keywords: Coincidence detection; Voltage threshold; Coherent activity; Temporal coding; Rate coding; Integrate-andfire neuron

\section{Introduction}

The issue of how neurons read out the temporal structure of the input and how they transform this structure into a firing rate pattern has been addressed by several authors and is attracting an increasing amount of interest. König et al. (1996) have argued that the main prerequisite for coincidence detectors is that the mean interspike interval is long as compared to the integration time

\footnotetext{
* Corresponding author.

E-mail: kempter@physik.tu-muenchen.de
}

which neurons need to effectively sum synaptic potentials. The importance of the effective (membrane) time constant of neurons has also been emphasized by Softky (1994). In addition, Abeles (1982) has shown that the value of the spike threshold and the number of synapses are relevant parameters as well.

In summary, some general principles have already been outlined, but not verified, and a detailed investigation of conditions under which neurons can act as coincidence detectors is not available yet. In the following we will substantiate some of the above statements and show explicitly 
the dependence of the precision of neuronal coincidence detection upon the membrane time constant, the input statistics, and the voltage threshold at which an action potential is generated. Specifically, we want to tackle the question of whether and to what extent a neuron that receives periodically modulated input with noise can detect the degree of synchrony and convert a time-coded signal into a rate-coded one. Here we concentrate on the essentials, in particular, on the dependence of the neurons behavior upon the threshold. For a more comprehensive and mathematically detailed account the reader is referred to Kempter et al. (1998).

\section{Neuron model and temporal coding of the input}

We study a single integrate-and-fire (I \& F) unit that receives stochastic input spike trains through $N$ independent channels. The neuron fires, if its membrane potential $u(t)$ approaches the threshold $\vartheta$ from below. This defines a firing time $t^{n}$ with integer $n$. After an output spike, which need not be described explicitly, the membrane potential is reset to 0. Between two firing events the membrane voltage changes according to the linear differential equation

$\frac{\mathrm{d}}{\mathrm{d} t} u(t)=-\frac{1}{\tau_{m}} u(t)+i(t)$

where $i$ is the total input current and $\tau_{m}>0$ the membrane time constant. The input is due to presynaptic activity. The spike arrival times at a given synapse $j$, with $1 \leq j \leq N$, are labeled by $t_{j}^{f}$ where $f=1,2, \ldots$ is a running spike index.

Each presynaptic spike evokes a small postsynaptic current (PSC) which decays exponentially with time constant $\tau_{s}>0$. All synapses are equal in the sense that the incoming spikes evoke PSCs of identical shape and amplitude. The total input of the neuron is then taken to be $i(t)=\tau_{s}^{-1} \Sigma_{j=1}^{N}$ $\Sigma_{f} \exp \left[-\left(t-t_{j}^{f}\right) / \tau_{s}\right] \theta\left(t-t_{j}^{f}\right)$ where $\theta($.$) denotes$ the Heaviside step function with $\theta(s)=0$ for $s \leq 0$ and $\theta(s)=1$ for $s>0$. We substitute the total input in Eq. (1) and integrate. This yields the membrane potential at the hillock (Gerstner et al. 1996a)

$$
u(t)=\sum_{j=1}^{N}\left[\sum_{f} \epsilon\left(t-t_{j}^{f}\right)\right]+\sum_{n} \eta\left(t-t^{n}\right)
$$

The first term on the right in Eq. (2),

$\epsilon(s)=\frac{\tau_{m}}{\tau_{m}-\tau_{s}}\left[\exp \left(-\frac{s}{\tau_{m}}\right)-\exp \left(-\frac{s}{\tau_{s}}\right)\right] \theta(s)$

describes the typical time course of an excitatory postsynaptic potential (EPSP). If $\tau_{s}=\tau_{m}$, we have instead of Eq. (3) the so-called alpha function, $\epsilon(s)=\left(s / \tau_{m}\right) \exp \left(-s / \tau_{m}\right) \theta(s)$. The second contribution to Eq. (2), $\eta(s)=-\vartheta \exp \left(-s / \tau_{m}\right) \theta(s)$, accounts for the reset of the membrane potential after each output spike and incorporates neuronal refractoriness.

Input spikes are generated stochastically and arrive at each synapse $1 \leq j \leq N$ with $N \gg 1$ with a time-dependent $T$-periodic rate $\lambda^{\text {in }}(t)=\lambda^{\text {in }}(t+$ $T) \geq 0$. The probability of having a spike in the interval $(t, t+\Delta t)$ is $\lambda^{\text {in }}(t) \Delta t$ as $\Delta t \rightarrow 0$. In this way we obtain a nonstationary or inhomogenous Poisson process (cf. Kempter et al. (1998)) where input spikes are more or less phase-locked to a $T$-periodic stimulus. This kind of input is a temporal code. The average number of spikes that arrive during one period at a synapse will be called $p$. The time-averaged mean input rate is $\bar{\lambda}^{\text {in }}:=1 / T \int_{t_{0}}^{t_{0}+T} \mathrm{~d} t^{\prime} \lambda^{\text {in }}\left(t^{\prime}\right)$ and equals $p / T$.

To parameterize the input, we take the function

$\lambda^{\mathrm{in}}(t):=p \sum_{m=-\infty}^{\infty} G_{\sigma}(t-m T)$

where $G_{\sigma}($.) denotes a normalized Gaussian distribution with zero mean and standard deviation $\sigma>0$. The I \& F neuron receives input input from $N \gg 1$ presynaptic terminals. At each input terminal spikes arrive with a probability density given by Eq. (4). Note that spikes arriving at different synapses are generated independently. Moreover, input spike trains have no temporal memory and hence do not contain refractoriness.

The standard deviation $\sigma \in(0, \infty)$ of the Gaussian distribution in Eq. (4) can be used to characterize the degree of synchrony of the input. In the case $\sigma=0$, the input spikes arrive perfectly phaselocked and occur only at the times $t_{m}=m T$ with integer $m$, and the number of spikes arriving at time $t_{m}$ has a Poissonian distribution with 
parameter $p$. Instead of $\sigma$, we often consider another measure of synchrony, the so-called vector strength $r^{\text {in }}$ (Goldberg and Brown 1969). This measure can be defined as the amplitude of the first Fourier component of the periodic rate $\lambda^{\text {in }}(t)$ divided by the Fourier component of order zero. For the input in Eq. (4) we find $r^{\text {in }}=\exp [-(2 \pi /$ $T)^{2} \sigma^{2} / 2$ ]. By construction, we have $0 \leq r^{\text {in }} \leq 1$.

\section{Analysis of coincidence detection}

We study the coincidence detection properties of an I \& F unit with $N$ independent periodic inputs as introduced above. Specifically, we examine the influence of the membrane time constant $\tau_{m}$ and of the threshold value $\vartheta$ on the neurons capability to act as a coincidence detector.

In a standard firing rate description a neuron would be characterized by its gain function which describes the dependence of the output firing rate on the mean input rate. Such an approach is certainly possible for most biological neurons. For I \& F neurons this property has been studied by Stemmler (1996) and Troyer and Miller (1997). In the present paper, we go at least one step further. We vary the input under the constraint of a constant mean input rate, $\bar{\lambda}^{\text {in }}=$ const. The assumption of a constant mean input is not a limitation of our approach but a simplification which we make here in order to illustrate our main point. We want to show that even with constant mean input rate the mean output rate $\bar{\lambda}^{\text {out }}$ varies as a function of the temporal structure of the input, parameterized, e.g. by $r$ in . In other words, the neuron can 'read out' a temporal code. This property is essential for coincidence detection.

\subsection{Membrane time constant}

The membrane time constant $\tau_{m}$ has an important influence on the coincidence detection properties of an I \& F unit (Softky 1994; König et al. 1996). This is most easily seen in the limit $\tau_{m} \rightarrow \infty$. We are left with a simple nonleaky integrator; cf. Eq. (1). In this case, the mean output rate can be calculated explicitly. Integrating Eq. (1) from the $n$th output spike at $t^{n}$ to the next one at $t^{n+1}$ we obtain $\vartheta=\int_{t^{n}}^{t^{n+1}} \mathrm{~d} t i(t)$. A summation over $M$ spikes yields:

$\vartheta=\frac{t^{n+M}-t^{n}}{M} N \bar{\lambda}^{\mathrm{in}}+\frac{1}{M} \int_{t^{n}}^{t^{n+M}} \mathrm{~d} t\left[i(t)-N \bar{\lambda}^{\mathrm{in}}\right]$

where we have separated the right-hand side into a first term that represents the contribution of the mean input current $N \bar{\lambda}^{\text {in }}$ and a second term that is the fluctuation around the mean. In order to calculate the mean output rate $\bar{\lambda}^{\text {out }}$, we have to consider the limit $M \rightarrow \infty$. We introduce the mean output rate by defining $\bar{\lambda}^{\text {out }}:=\lim _{M \rightarrow \infty} M /\left(t^{n+M}\right.$ $\left.-t^{n}\right)$. As $M \rightarrow \infty$, the contribution from the second term in the right-hand side of Eq. (5) vanishes and we are left with $\bar{\lambda}^{\text {out }}=N \bar{\lambda}^{\text {in }} / \vartheta$. The mean output rate is independent of the explicit form of the time-dependent input rate $\lambda^{\text {in }}(t)$, especially $r^{\text {in }}$.

The limit of $\tau_{m} \rightarrow \infty$ is, of course, an abstraction. The above consideration holds, however, whenever the membrane time constant $\tau_{m}$ is much longer than the periodicity $T$ of the input, and if we have a 'low' threshold. In Fig. 1a we have demonstrated this scenario graphically for the case $\tau_{m}=5 T$. The interspike intervals (and hence the mean firing rate) do not differ significantly between the case of coherent input ( $r^{\text {in }}=1$; solid line) and the case of incoherent input $\left(r^{\text {in }}=0\right.$; dashed line).

On the other hand, if $\tau_{m}=T / 2$ as in Fig. $1 \mathrm{~b}$ and if we use the same threshold as in Fig. 1a, then the interspike intervals are much shorter for the case $r^{\text {in }}=1$ (solid line) than for the case $r^{\text {in }}=0$ (dashed line). Hence a 'small' membrane time constant $\tau_{m}$ (thus, a leaky integrator) is a necessary condition for a coincidence detector whose rate varies with $r^{\text {in }}$. But what is meant by a 'small' $\tau_{m}$ ? The answer depends on the value of the threshold $\vartheta$. The relevance of the parameter $\vartheta$ is discussed in the next paragraph.

\subsection{Voltage threshold}

We want to find a set of parameters which makes the neuron a good coincidence detector. What would be an optimal choice for the threshold parameter $\vartheta$ ? Let us assume for the 
a)

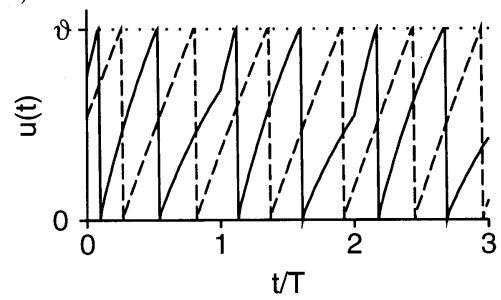

b)

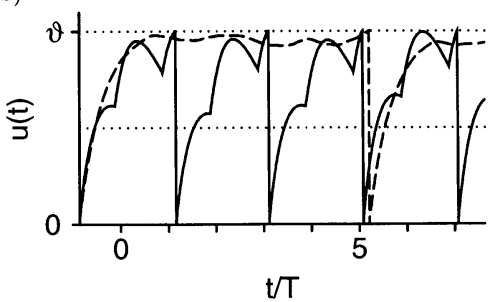

Fig. 1. Membrane potential $u$ of an I \& F neuron as a function of time $t$. The time constant of the synaptic input current is $\tau_{s}=T$. The threshold is $\vartheta=100$. The neuron receives input from 400 synapses with noise parameter $p=0.5$ and vector strength $r^{\text {in }}=1$ (perfectly phase-locked input: solid lines) and $r^{\text {in }}=0$ (completely random input: dashed lines); cf. Eq. (4). Whatever $r^{\text {in }}$, there is always a random component in the input. Even $r^{\text {in }}=1$ yields non-periodic responses because the number of input spikes at times $m T$ (with integer $m$ ) is Poisson distributed. (a) For a large membrane time constant $\tau_{m}=5 T$ the intervals between output spikes are nearly independent from the vector strength in the input. Such a neuron is a 'bad' coincidence detector. (b) For a small time constant $\tau_{m}=T / 2$ the mean interval between output spikes does depend on the vector strength in the input, if the threshold is chosen appropriately, e.g. $\vartheta=\bar{u}_{\infty}=100$ (upper dotted line). For a threshold at $\bar{u}_{\infty} / 2$ (lower dotted line), the first spike would have occurred, nearly independently of the input vector strength $r^{\text {in }}$, somewhere near $t=-T / 2$, whereas for a threshold at $\bar{u}_{\infty}$ (upper dotted line) the time to the first spike depends strongly on $r^{\text {in }}$. For $r^{\text {in }}=1$ (solid line) the first spike appears near $t=T$ and a second spike near $t=3 T$. For $r^{\text {in }}=0$ (dashed line) the first output spike occurs near $t=5 T$.

moment that the firing threshold is very high (formally, $\vartheta \rightarrow \infty$ ) and let us focus on the temporal behavior of the membrane voltage $u(t)$ with some input current $i$. The membrane potential cannot reach the threshold so that there is neither an output spike nor a reset to baseline, and the membrane voltage fluctuates around the mean voltage $\bar{u}_{\infty}=N \bar{\lambda}^{i n} \tau_{m}$; see also Fig. 1b. (The voltage $\bar{u}_{\infty}$ equals $u(t)$ as $t \rightarrow \infty$, provided the total input current is equal to its mean value $i=N \bar{\lambda}^{\text {in }}$.) We now lower the threshold so that the neuron occasionally emits a spike. The coincidence detection properties of this neuron depend on the location of $\vartheta$ relative to $\bar{u}_{\infty}$.

In Fig. 2a we show the dependence of the output firing rate upon the input vector strength $r^{\text {in }}$ for various values of the threshold $\vartheta$. If the threshold is low, the mean output firing rate $\bar{\lambda}^{\text {out }}$ is rather insensitive to the input vector strength $r^{\text {in }}$ for $\vartheta<\bar{u}_{\infty}$ (consider the top curve in Fig. 2a labeled $\vartheta_{-1}$ ). We therefore get a poorly performing coincidence detector. In contrast, a threshold $\vartheta>\bar{u}_{\infty}$ (e.g. $\vartheta_{1}$ or $\vartheta_{2}$ ) leads to a large variation of the mean output firing rate as a function of the input vector strength, $\bar{\lambda}^{\text {out }}\left(r^{\text {in }}\right)$. Consequently we seem to obtain a better coincidence detector.

In order to understand the mechanism of this improvement, let us return to Fig. 1b. The trajec- tory of the membrane voltage $u(t)$ is shown for two cases, viz. for random and phase-locked input. Let us imagine a threshold $\vartheta$ well below $\bar{u}_{\infty}$, say at $\bar{u}_{\infty} / 2$. In this case the spike following the reset at $t \approx-0.75$ is triggered after a short time interval, the length of which depends only marginally on the degree of synchrony in the input. We are close to the regime of a nonleaky integrator. Formally, this can be seen from Eq. (1) using an argument along the following lines. Between two firings the membrane potential always stays below threshold, $u(t)<\vartheta$. If the average current is much larger than $\vartheta / \tau_{m}$, then the first term in the right-hand side of Eq. (1) can be neglected and we do have a nonleaky neuron. In contrast to that, let us consider the case $\vartheta>\bar{u}_{\infty}$. The threshold $\vartheta$ can be reached only if the fluctuations of $u(t)$ are large enough.

The fluctuations consist of a statistic contribution, due to spike input (shot noise), and periodic oscillations, due to phase-locked (coherent) input. The key observation is that with increasing synchrony in the input the periodic oscillations get larger and therefore the output firing rate increases. In order to quantify this effect we introduce a new parameter $E$, the coherence gain:

$E\left(r^{\text {in }}\right):=\frac{\bar{\lambda}^{\text {out }}\left(r^{\text {in }}\right)}{\bar{\lambda}^{\text {out }}(0)}$ 

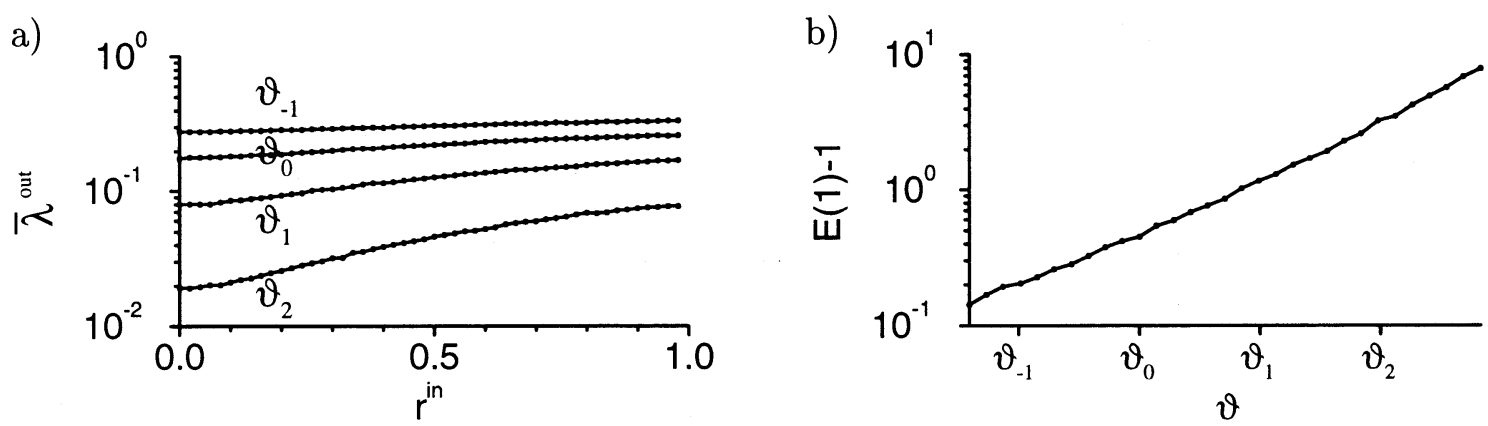

Fig. 2. Coincidence detection properties depend on the input statistics and the value of the spiking threshold. (a) Mean output firing rate $\bar{\lambda}^{\text {out }}$ (in units of spikes per period $T$ ) as a function of the input vector strength $r^{\text {in }}$ for four different values of the threshold, $\vartheta_{-1}=\bar{u}_{\infty}-\Delta u, \vartheta_{0}=\bar{u}_{\infty}, \vartheta_{1}=\bar{u}_{\infty}+\Delta u, \vartheta_{2}=\bar{u}_{\infty}+2 \Delta u$. In short, $\vartheta_{1}=\bar{u}_{\infty}+l \Delta u$, where $l \in\{-1,0,1,2\}$. The mean voltage is $\bar{u}_{\infty}=200$ and $\Delta u=10 / \sqrt{2}$. For large $\vartheta\left(\right.$ e.g. $\left.\vartheta_{2}\right)$ the output rate varies by an order of magnitude if the temporal coherence of the input increases from $r^{\text {in }}=0$ to $r^{\text {in }}=1$. On the other hand, for $\vartheta=\vartheta_{-1}$ the rate $\bar{\lambda}^{\text {out }}$ hardly depends on the temporal structure of the input. With $r^{\text {in }}$ fixed, the rate $\bar{\lambda}^{\text {out }}$ increases with decreasing $\vartheta$ in all cases; (b) $E(1)-1$ is plotted against the threshold $\vartheta$. The coherence gain $E$ has been defined in Eq. (6). Simulation parameters in a and b: $\tau_{m}=\tau_{s}=T, N p=200$. Data points have been obtained by measuring time intervals that were needed to produce $10^{4}$ output spikes.

For example, $E(1)$ is the ratio between the mean firing rate for fully coherent input and the rate for completely incoherent input. For a visualization of $E(1)$ consider Fig. 2a. For each curve in Fig. $2 \mathrm{a}, E(1)$ is the ratio between between the rightmost and the leftmost value.

A coherence gain $E\left(r^{\text {in }}\right) \approx 1$ means that the I \& F neuron does not operate as a coincidence detector, whereas $E\left(r^{\text {in }}\right) \gg 1$ hints at good coincidence detection. With the definition of the coherence gain, the four graphs in Fig. 2a can be summarized by Fig. 2b, where the dependence of $E\left(r^{\text {in }}\right)$ upon the threshold is shown for the special case $r^{\text {in }}=1$. The coherence gain $E(1)$ increases with increasing threshold $\vartheta$.

The output of a coincidence detector must convey a signal concerning the nature of the input (coherent or not coherent) in a finite amount of time. Let us consider an interval of duration $I$. Even neurons with $E(1) \gg 1$ are basically useless as coincidence detectors, if their firing rate is so low that there is most likely not a single spike in I. This is the case, e.g. for leaky I \& F units with a voltage threshold $\vartheta$ well above $\bar{u}_{\infty}$ and $I$ of the order of a few $T$ (cf. Fig. $2 \mathrm{a}, \vartheta=\vartheta_{2}$ ). As may be seen from the sequence of curves from Fig. 2a, the mean output rate decreases rapidly with increasing threshold (note the logarithmic scale).
The other extreme case is a high mean output rate which implies a threshold well below $\bar{u}_{\infty}$ (cf. Fig. $2, \vartheta=\vartheta_{-1}$ ). In this regime $E(1)$ is low-we are in the regime of a nonleaky integrator where neurons are not able to perform a coincidence detection either (cf. Fig. 1a). Between the above two limits there is an intermediate regime with an optimal threshold for coincidence detection. It is 'optimal' in the sense that both the spike rate and the coherence gain are high.

Since the input is noisy, the number $n$ of output spikes in a finite interval $I$, from which the rate has to be estimated, is a random quantity. We assume that $n$ is Poisson distributed around a

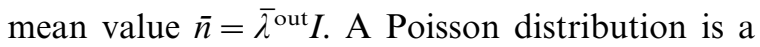
good approximation if spiking is driven by the variance of the neuron's input; see, e.g. Troyer and Miller (1997). For two different input vector strengths, e.g. $r^{\text {in }}=0$ and $r^{\text {in }}=\hat{r}^{\text {in }}>0$, we have two different distributions $P\left(r^{\text {in }}\right)$ with mean values $\bar{n}(0)$ and $\bar{n}\left(\hat{r}^{\text {in }}\right)$, respectively. The task is to distinguish the two cases based on a single measurement of the number of output spikes in $I$. We therefore need a number $n^{\prime}$ as a decision boundary. If $n \geq n^{\prime}$, then we classify the input to have $r^{i n}=\hat{r}^{i n}>0$; if $n<n^{\prime}$, then we classify $r^{\text {in }}=$ 0 . Clearly, the probability for the correct decision depends on $n^{\prime}$. In general, the optimal choice for the decision boundary $n_{\text {opt }}^{\prime}$ is the point where the 
two spike distributions $P\left(r^{\text {in }}=0\right)$ and $P\left(r^{\text {in }}=\hat{r}^{\text {in }}\right)$ cross; see, e.g. Duda and Hart (1973).

A reliable decision is impossible, if the two distributions $P\left(\hat{r}^{\mathrm{in}}\right)$ and $P(0)$ have a large overlap. In order to quantify the discriminability of the two distributions we consider their normalized 'distance':

$\gamma:=\frac{\bar{n}\left(r^{\text {in }}\right)-\bar{n}(0)}{\sqrt{\bar{n}\left(r^{\text {in }}\right)}+\sqrt{\bar{n}(0)}}=\sqrt{\bar{n}\left(r^{\text {in }}\right)}-\sqrt{\bar{n}(0)}$.

We call $\gamma$ the quality factor for coincidence detection. It is obtained by dividing the difference of the mean values by the sum of their standard deviations. Using the definition of the coherence gain $E$ in Eq. (6) we obtain from Eq. (7)

$\gamma=\sqrt{I \bar{\lambda}^{\text {out }}(0)}\left[\sqrt{E\left(r^{\text {in }}\right)-1}\right]$

Eq. (8) shows how the quality factor $\gamma$ increases with increasing $I, \bar{\lambda}^{\text {out }}(0)$, and $E\left(r^{\text {in }}\right)$. It is important to realize that $\bar{\lambda}^{\text {out }}(0)$ and $E\left(r^{\text {in }}\right)$ are not independent variables. To classify the quality of coincidence detection, $\gamma$ is a suitable measure. More complicated quantities like 'mutual information between the spike counts and the presence or absence of the periodic input' and the 'probability of correct detection in the discrimination between the two alternatives' (Stemmler 1996) can be expanded in powers of $\gamma$.

The graphs in Fig. 3 show that there is an optimal choice for the threshold that maximizes $\gamma$. Each curve shows the quality factor $\gamma$ as a function of the threshold $\vartheta$. We argue that $\gamma(\vartheta)$ approaches zero for $\vartheta \rightarrow 0$ and also for $\vartheta \rightarrow \infty$. Thus there must be at least one maximum in between. The case $\vartheta \rightarrow 0$ corresponds to an infinitely high membrane time constant, as we have discussed before. This means that the neuron is effectively a nonleaky integrator. For this kind of integrator, the mean output rate does not depend on the input structure. Thus, $E=1$ and $\gamma(\vartheta) \rightarrow 0$ as $\vartheta \rightarrow 0$ (cf. Eq. (8)). In the case $\vartheta \rightarrow \infty$ spiking becomes a rare event. Hence $\bar{n}\left(r^{\text {in }}\right) \rightarrow 0$ for $\vartheta \rightarrow \infty$. Since $\bar{n}\left(r^{\text {in }}\right)>\bar{n}(0)$ it follows from Eq. (7) that $\gamma \rightarrow 0$ as $\vartheta \rightarrow \infty$. Note that $\gamma$ exhibits a broad maximum and therefore depends only weakly on the threshold. Thus, the exact value of the threshold is not a critical parameter for coincidence detection. In contrast to that, $\gamma$ varies strongly, if we change, e.g. the neuronal time constants $\tau_{m}$ and $\tau_{s}$, the number $N$ of synapses, or the number $p$ of inputs spikes per period $T$, where $p / T=\bar{\lambda}^{\text {in }}$ (Fig. 3; see also Kempter et al. 1998).

\section{Discussion}

The membrane time constant (Fig. 1), the statistics of the input (Fig. 2), and the threshold (Figs. 2 and 3) of an I \& F neuron influence the capability of the neuron to work as a coincidence detector for periodically modulated spike input. Different parameter regimes have been compared in terms of the quality factor $\gamma$ for coincidence detection. As a function of $\vartheta$, the quality factor $\gamma$

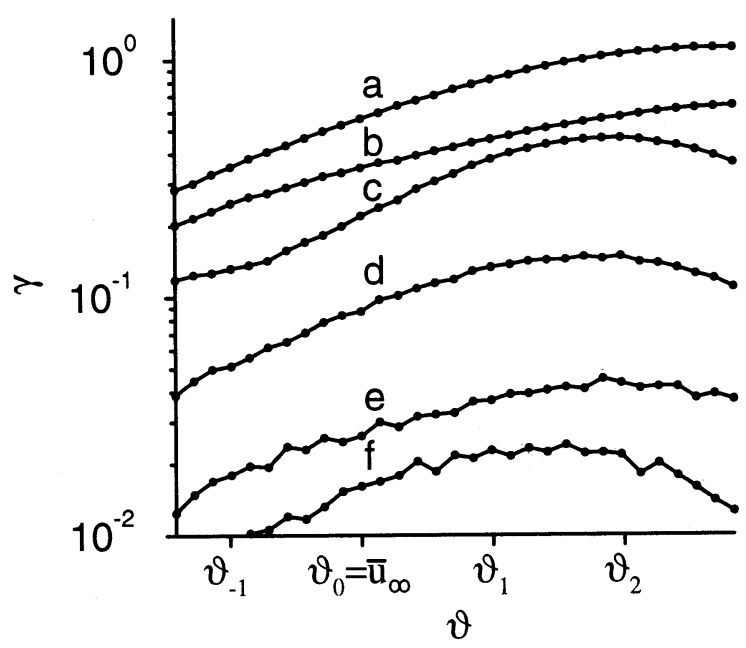

Fig. 3. Coincidence detection properties depend on the value of the spiking threshold for different scenarios (a)-(f) of simulation parameters. The parameter $\gamma$ in units of $T^{-1 / 2} I^{1 / 2}$ (cf. Eq. (8)) indicates the quality of a coincidence detector. It shows a maximum above the mean voltage $\bar{u}_{\infty}=\vartheta_{0}$; numerical values for $\vartheta_{-1}, \vartheta_{0}, \vartheta_{1}$, and $\vartheta_{2}$ as in Fig. 2. In practical situations it is immaterial, though, whether we take, e.g. $\vartheta_{1}$ or $\vartheta_{2}$. That is to say, the choice of the threshold is not critical because there is a broad maximum. Simulation parameters: (a) $\tau_{m}=\tau_{s}=T / 4, N p=800$; (b) $\tau_{m}=\tau_{s}=T / 2, N p=400$; (c) $\tau_{m}=$ $T / 2, \tau_{s}=T, N p=400$; (d) $\tau_{m}=\tau_{s}=T, N p=200$; (e) $\tau_{m}=2 T$, $\tau_{s}=T, N p=100$; (f) $\tau_{m}=\tau_{s}=2 T, N p=100$. Parameters have been chosen so that $\bar{u}_{\infty}=N p \tau_{m} / T=200$ is constant, thus simplifying the comparison. Each point corresponds to $10^{4}$ output spikes. 
shows a maximum which suggests that there is an optimal threshold value $\vartheta_{\text {opt }}$ for coincidence detection (Fig. 3). For optimal coincidence detection the threshold has to exceed the mean membrane voltage $\bar{u}_{\infty}=N \bar{\lambda}^{\mathrm{in}} \tau_{m}$ of a neuron $\left(\vartheta_{\mathrm{opt}}>\bar{u}_{\infty}\right)$. A high threshold $\left(\vartheta \gg \vartheta_{\text {opt }}\right)$ implies a low mean output firing rate which destroys the advantage of a high coherence gain $E$. A low threshold $\left(\vartheta \ll \vartheta_{\text {opt }}\right)$ leads to the regime of a nonleaky integrator which is not at all suited to coincidence detection.

Coincidence detection in 'real' neurons requires an adaptive mechanism to control the threshold. There are several different possibilities for doing so. First, we could imagine a control loop which adjusts the threshold in the appropriate regime. This might be difficult to implement, but could be achieved, for example, if each spike is followed by long-lasting hyperpolarizing afterpotential. Alternatively, we could envisage a feedback loop of inhibitory neurons that adjust the mean input. A control of input strength is also possible through synaptic changes (Abbott et al. 1997; Tsodyks and Markram 1997). Finally, it has been shown in a model study (Gerstner et al. 1996b) that potentiation and depression of synaptic weights can balance each other so that the effective input strength is always normalized; see also Markram et al. (1997). However, the threshold is not a critical parameter for coincidence detection, as is illustrated by the broad maximum of $\gamma$ as a function of the threshold in Fig. 3. The threshold also determines the mean firing rate of a neuron. For reasonable firing rates the quality factor remains of the same order of magnitude as its maximum value.

Low mean firing rates, e.g. of cortical neurons with rates of the order of a few Hertz, may serve to keep energy consumption low. Generation and transmission of action potentials is metabolically expensive. Minimization of the mean output firing rate without significantly lowering the quality factor hardly affects function but saves energy.

The phenomena discussed in this paper are intimately connected to stochastic resonance (Wiesenfeld and Moss 1995). In stochastic resonance theory, the level of noise is is considered as a free parameter and optimized so as to improve the quality of signal transmission. With view to biological systems, it is, however, questionable whether neurons are able to change the level of noise in their input. On the other hand, a neuron has potentially the chance of adapting its threshold to an optimal value, as discussed in this section. We emphasize that the optimal threshold for coincidence detection is, similarly to stochastic resonance, always above $\bar{u}_{\infty}$. Further investigations demonstrate that the optimal threshold is above $\bar{u}_{\infty}$ by an amount which of the same order of magnitude as the noise amplitude of the membrane potential (Kempter et al. 1998).

Even if we know $\gamma$, we should be be careful and avoid rash conclusions about a neuron's task. As an illustration, let us consider a neuron whose $\gamma$ is small. One might argue that such a neuron cannot function as a coincidence detector, and this is certainly correct if we consider the neuron as a single unit. But if there is a pool neurons operating in the same pathway and receiving the same type of input, the output of all these neurons together could provide a secure cue for a decision (Spiridon et al. 1998). Similarly, the waiting time which is necessary to make a correct decision with high reliability can be reduced by using a pool neurons. That is to say, the following two counting methods are equivalent: a system can either use the output spike count of a single neuron in an interval $I$ or counting the number of spikes of $L$ statistically independent, identical neurons operating in the same pathway during a period of time $I / L$. Weak correlations from common inputs rescale the absolute values but do not alter the conclusion. Inhibitory connections between the neurons lead to anti-correlations and can help to increase the signal-to-noise ratio and hence to shorten the waiting time (Spiridon et al. 1998).

\section{Acknowledgements}

This work has been supported by the Deutsche Forschungsgemeinschaft (DFG) under grant numbers He 1729/8-1 (RK) and He 1729/2-2, 8-1 (WG). 


\section{References}

Abbott, L.F., Varela, J.A., Sen, K., Nelson, S.B., 1997. Synaptic depression and cortical gain control. Science 275, 220-224.

Abeles, M., 1982. Role of the cortical neuron: integrator or coincidence detector? Isr. J. Med. Sci. 18, 83-92.

Duda, R.O., Hart, P.E., 1973. Pattern Classification and Scene Analysis. Wiley, New York.

Gerstner, W., van Hemmen, J.L., Cowan, J.D., 1996a. What matters in neuronal locking? Neural. Comput. 8, 1689-1712.

Gerstner, W., Kempter, R., van Hemmen, J.L., Wagner, H., 1996b. A neuronal learning rule for sub-millisecond temporal coding. Nature 383, 76-78.

Goldberg, J.M., Brown, P.B., 1969. Response of binaural neurons of dog superior olivary complex to dichotic tonal stimuli: some physiological mechanisms of sound localization. J. Neurophysiol. 32, 613-636.

Kempter, R., Gerstner, W., van Hemmen, J.L., Wagner, H., 1998. Extracting oscillations: neuronal coincidence detection with noisy periodic spike input, Neural Comput. $101987-$ 2017.

König, P., Engel, A.K., Singer, W., 1996. Integrator or coincidence detector? The role of the cortical neuron revisited. Trends Neurosci. 19, 130-137.
Markram, H., Lübke, J., Frotscher, M., Sakmann, B., 1997. Regulation of synaptic efficacy by coincidence of postsynaptic APs and EPSPs. Science 275, 213-215.

Softky, W., 1994. Sub-millisecond coincidence detection in active dendritic trees. Neuroscience 58, 13-41.

Spiridon, M., Chow, C.C., Gerstner, W., 1998. Frequency spectrum of coupled stochastic neurons with refractoriness. In: Niklasson, L., Bodem, M., Zienke, T. (Eds.), Proceedings of the Eighth International Conference on Artificial Neural Networks (ICANN '98). Springer, Berlin, pp. 337342 .

Stemmler, M., 1996. A single spike suffices: the simplest form of stochastic resonance. Network 7, 687-716.

Troyer, T.W., Miller, K.D., 1997. Physiological gain leads to high ISI variability in a simple model of a cortical regular spiking cell. Neural Comput. 9, 971-983.

Tsodyks, M.V., Markram, H., 1997. The neural code between neocortical pyramidal neurons depends on neurotransmitter release probability. Proc. Natl. Acad. Sci. USA 94, $719-723$.

Wiesenfeld, K., Moss, F., 1995. Stochastic resonance and the benefits of noise: from ice ages to crayfish and SQUIDs. Nature 373, 33-36. 